\title{
Electronic Structure and Enhanced Charge-Density Wave Order of Monolayer VSe $\mathrm{V}_{2}$
}

\author{
Jiagui Feng, ${ }^{1,2, *}$ Deepnarayan Biswas,,${ }^{1, *}$ Akhil Rajan,,${ }^{1, *}$ Matthew D. Watson, ${ }^{1}$ Federico Mazzola, ${ }^{1}$ \\ Oliver J. Clark, ${ }^{1}$ Kaycee Underwood, ${ }^{1}$ Igor Marković, ${ }^{1,3}$ Martin McLaren, ${ }^{1}$ Andrew Hunter, ${ }^{1}$ David M. Burn, ${ }^{4}$ \\ Liam B. Duffy, ${ }^{5,6}$ Sourabh Barua, ${ }^{7,} \dagger$ Geetha Balakrishnan, ${ }^{7}$ François Bertran, ${ }^{8}$ Patrick Le Fèvre, ${ }^{8}$ \\ Timur K. Kim, ${ }^{9}$ Gerrit van der Laan, ${ }^{4}$ Thorsten Hesjedal, ${ }^{5}$ Peter Wahl, ${ }^{1}$ and Phil D. C. King ${ }^{1, \ddagger}$ \\ ${ }^{1}$ SUPA, School of Physics and Astronomy, University of St. Andrews, St. Andrews KY16 9SS, United Kingdom \\ ${ }^{2}$ Suzhou Institute of Nano-Tech. and Nanobionics (SINANO), \\ CAS, 398 Ruoshui Road, SEID, SIP, Suzhou, 215123, China \\ ${ }^{3}$ Max Planck Institute for Chemical Physics of Solids, Nöthnitzer Straße 40, 01187 Dresden, Germany \\ ${ }^{4}$ Magnetic Spectroscopy Group, Diamond Light Source, Didcot, OX11 ODE, United Kingdom \\ ${ }^{5}$ Department of Physics, University of Oxford, Oxford, OX1 3PU, United Kingdom \\ ${ }^{6}$ ISIS, STFC, Rutherford Appleton Laboratory, Didcot, OX11 OQX, United Kingdom \\ ${ }^{7}$ Department of Physics, University of Warwick, Coventry CV4 7AL, United Kingdom \\ ${ }^{8}$ Synchrotron SOLEIL, CNRS-CEA, L'Orme des Merisiers, Saint-Aubin-BP48, 91192 Gif-sur-Yvette, France \\ ${ }^{9}$ Diamond Light Source, Harwell Campus, Didcot, OX11 ODE, United Kingdom
}

\begin{abstract}
How the interacting electronic states and phases of layered transition-metal dichalcogenides evolve when thinned to the single-layer limit is a key open question in the study of two-dimensional materials. Here, we use angle-resolved photoemission to investigate the electronic structure of monolayer $\mathrm{VSe}_{2}$ grown on bi-layer graphene/SiC. While the global electronic structure is similar to that of bulk $\mathrm{VSe}_{2}$, we show that, for the monolayer, pronounced energy gaps develop over the entire Fermi surface with decreasing temperature below $T_{c}=140 \pm 5 \mathrm{~K}$, concomitant with the emergence of charge-order superstructures evident in low-energy electron diffraction. These observations point to a charge-density wave instability in the monolayer which is strongly enhanced over that of the bulk. Moreover, our measurements of both the electronic structure and of x-ray magnetic circular dichroism reveal no signatures of a ferromagnetic ordering, in contrast to the results of a recent experimental study as well as expectations from density-functional theory. Our study thus points to a delicate balance that can be realised between competing interacting states and phases in monolayer transition-metal dichalcogenides.
\end{abstract}

Keywords: $\mathrm{VSe}_{2}$, charge-density wave, transition-metal dichalcogenide, monolayer

Control over materials thickness down to the singleatom scale has emerged as a powerful tuning parameter for manipulating not only the single-particle band structures of solids, but increasingly also their interacting electronic states and phases. ${ }^{1-7}$ A particularly attractive materials system in which to explore this is the transitionmetal dichalcogenides (TMDs), both because of their naturally-layered van der Waals structures as well as the wide variety of materials properties which they are known to host. ${ }^{4-13}$ These include much-studied charge-density wave $(\mathrm{CDW})$ states $^{14-18}$ which exhibit an intricate interplay with superconductivity in several TMDs. ${ }^{19-22}$ Yet, a consistent picture is still to emerge over how their chargeordered states evolve when reducing materials thickness down to a single monolayer. ${ }^{23-29}$

In part, this reflects an intrinsic competition, whereby the microscopic interactions which drive such phase formation and the fluctuations which destabilise it are both expected to become strengthened in the two-dimensional limit as compared to their bulk three-dimensional counterparts. Here, we investigate $\mathrm{VSe}_{2}$ as a particularly attractive candidate system in which to probe such phenomenology. It is known to host a CDW in the bulk, with an onset temperature of $T_{c} \approx 110 \mathrm{~K} .^{15,30}$ Despite its simple layered structure (Fig. 1(a)), the bulk order- ing wavevector $\mathbf{q}_{\mathrm{CDW}}=(1 / 4,1 / 4, \sim 1 / 3)$ has a significant component in the out-of-plane direction, ${ }^{15,30}$ which has been attributed to a nesting of the strongly threedimensional Fermi surface at this wavevector. ${ }^{31}$ Reducing the material thickness to a single monolayer (ML), where the electronic structure must become strictly twodimensional, could therefore be expected to have a major influence on the charge order in $\mathrm{VSe}_{2}$. Studies of multi-layer exfoliated flakes have so far indicated both a strengthening and suppression of its CDW with reducing thickness, ${ }^{23,32,33}$ necessitating further studies in the true monolayer limit.

Moreover, first-principles calculations have consistently predicted that a robust ferromagnetic order should emerge for monolayer $\mathrm{VSe}_{2}$, with a pronounced exchange splitting of the near- $E_{\mathrm{F}}$ electronic states in the $1 T$ polymorph of more than $500 \mathrm{meV} \cdot{ }^{32,34}$ A very recent experimental report that such a ferromagnetic state persists up to room temperature in monolayer $\mathrm{VSe}_{2}{ }^{35}$ raised the tantalising prospect that this system could form one of the emerging class of $2 \mathrm{D}$ magnets. ${ }^{36,37}$ There are, however, a number of critical unexplained features such as a reported magnetisation as high as $15 \mu_{\mathrm{B}}$ per vanadium, motivating further investigation of this putative ferromagnetic state. 
(a)

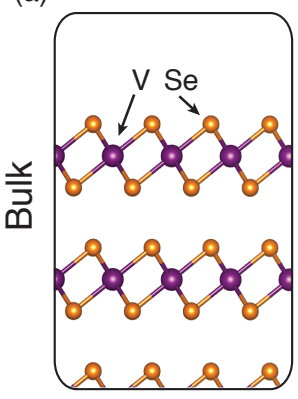

(f)

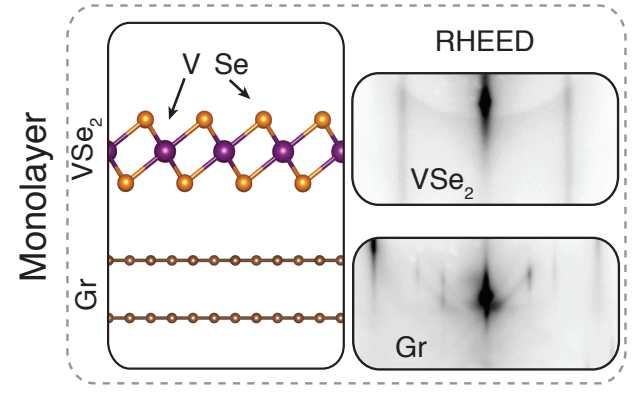

(c)

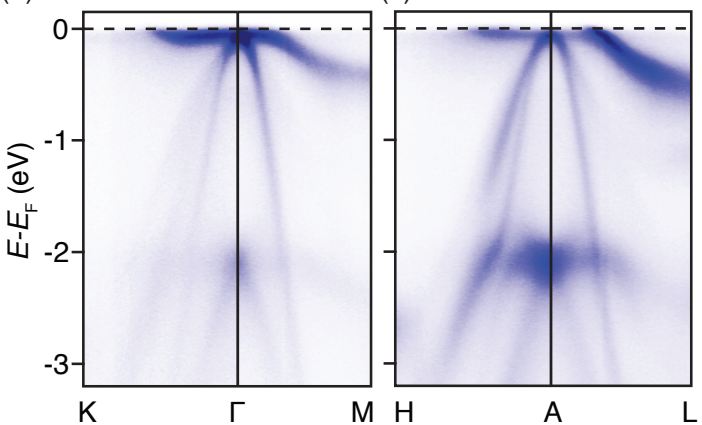

(h) (d)

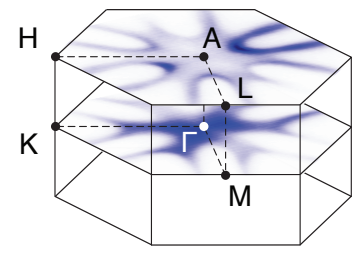

$\min \square \max$ (e)

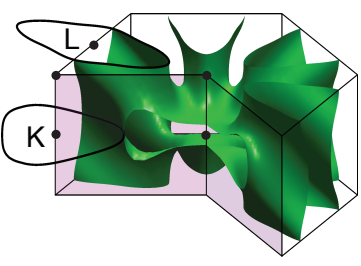

(j)

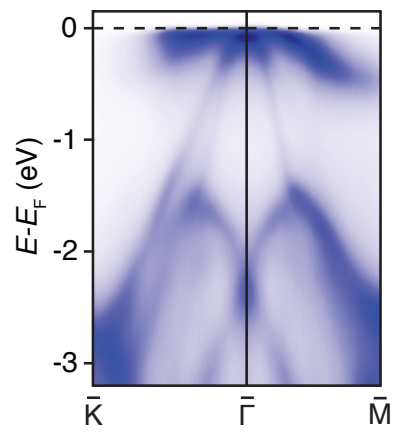

(i)
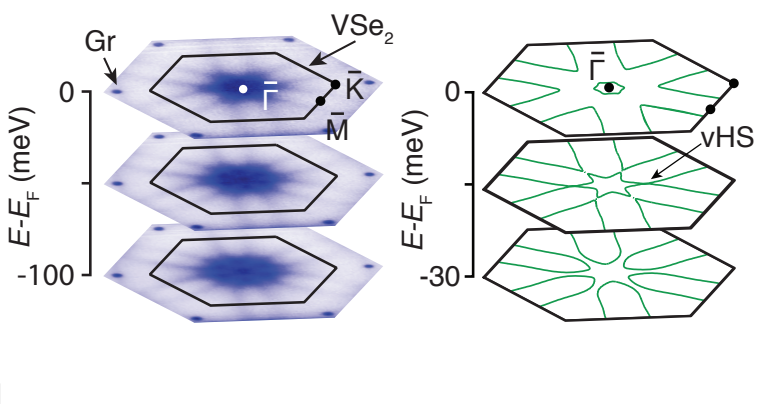

FIG. 1. Normal-state electronic structure of bulk and monolayer VSe $\mathrm{V}_{2}$. (a) $1 T$ crystal structure of bulk VSe 2 , and (b,c) its normal-state electronic structure $(T \approx 170 \mathrm{~K})$ measured along the $(\mathrm{b}) \mathrm{K}-\Gamma-\mathrm{M}\left(k_{z} \approx 0, h \nu=92.5 \mathrm{eV}\right)$ and $(\mathrm{c}) \mathrm{H}-\mathrm{A}-\mathrm{L}$ $\left(k_{z} \approx \pi / c, h \nu=110 \mathrm{eV}\right)$ directions of the bulk Brillouin zone. (d) Corresponding Fermi surfaces measured in the $\mathrm{K}-\Gamma-\mathrm{M}$ and $\mathrm{H}-\mathrm{A}-\mathrm{L}$ planes and (e) calculated DFT Fermi surface throughout the three-dimensional Brillouin zone . (f) Crystal structure of $\mathrm{VSe}_{2}$ /bilayer graphene ( $\mathrm{Gr}$ ) heterostructure and (g) RHEED measured along the [1100] direction of the graphene substrate (bottom) and following growth of $\mathrm{ML} \mathrm{VSe}_{2}$ (top). (h) Electronic structure $\left(T=170 \mathrm{~K}\right.$ ) of monolayer VSe $\mathrm{V}_{2}$ measured along the $\overline{\mathrm{K}}-\bar{\Gamma}-\overline{\mathrm{M}}$ direction $(h \nu=21.2 \mathrm{eV})$. (i,j) Corresponding (i) measured $(h \nu=110 \mathrm{eV})$ and (j) calculated Fermi surface and near- $E_{\mathrm{F}}$ constant energy contours of monolayer $\mathrm{VSe}_{2}$, revealing the close proximity of the van Hove singularity to the Fermi level.

In this work, we synthesise monolayer $\mathrm{VSe}_{2}$ films on both bilayer graphene/SiC and highly oriented pyrolytic graphite (HOPG) substrates using molecularbeam epitaxy (see Methods). We use both in situ and synchrotron-based angle-resolved photoemission spectroscopy (ARPES) to directly probe their temperaturedependent electronic structure, and search for ferromagnetic order from x-ray magnetic circular dichroism (XMCD). Our measurements indicate no ferromagnetism down to $10 \mathrm{~K}$. Instead they reveal that a charge-density wave which gaps the entire high-temperature Fermi surface is the dominant instability at low temperatures in monolayer $\mathrm{VSe}_{2}$.

Figure 1 summarises the normal-state electronic structure of bulk and monolayer $\mathrm{VSe}_{2}$ from our ARPES measurements of single crystal samples and epitaxial monolayers, respectively. Both the bulk and monolayer samples host a qualitatively similar electronic structure. The Fermi surface is formed from a relatively weaklydispersive $\mathrm{V}$ 3d-electron-derived band. ${ }^{38}$ An additional set of hole-like bands, derived from the Se $4 p$ orbitals, disperse steeply down from the $\Gamma$-point into the lower- lying valence bands, with their band maximum situated $65 \mathrm{meV}$ below $E_{\mathrm{F}}$.

In bulk, the Fermi surface of $\mathrm{VSe}_{2}$ is known to be three-dimensional. ${ }^{31}$ Consistent with this, we find subtle but important differences in the electronic structure measured using photon energies chosen to probe close to the centre and boundary of the Brillouin zone along $k_{z}$, respectively (Fig. 1(b-d)). In particular, the $\mathrm{V}$-derived state crosses $E_{\mathrm{F}}$ along the $\mathrm{A}-\mathrm{L}$ direction, leading to a Fermi surface in the $\mathrm{H}-\mathrm{A}-\mathrm{L}$ plane which forms closed electron-like contours of almost elliptical cross-section enclosing the zone-edge L-points. In contrast, this band does not quite reach $E_{\mathrm{F}}$ along the $\Gamma-\mathrm{M}$ direction, with the clear Fermi crossing along $\Gamma-\mathrm{K}$ instead forming the tip of a large trigonally-warped hole-like Fermi contour located around each corner K-point within the $\mathrm{K}-\Gamma-\mathrm{M}$ plane. This is in agreement with the calculated Fermi surface from density-functional theory (DFT, Fig. 1(e)) as well as previous soft x-ray ARPES measurements, ${ }^{31}$ which indicate the $k_{z}$-dependent evolution of the Fermi surface between these two distinct topologies.

In contrast, the Fermi surface of ML-VSe ${ }_{2}$ must be 
(a) $\mathrm{Gr} / \mathrm{SiC}$

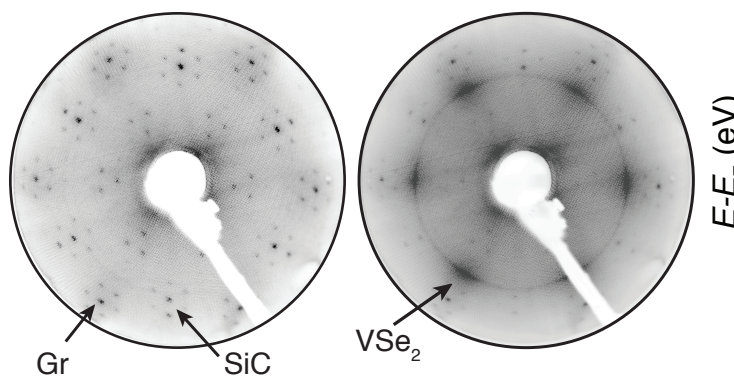

(c) $I_{\text {LEED }}(40 \mathrm{~K})-I_{\text {LEED }}(200 \mathrm{~K})$

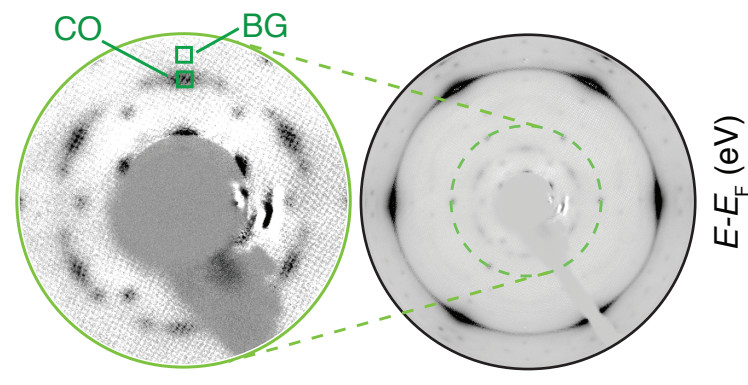

(d)

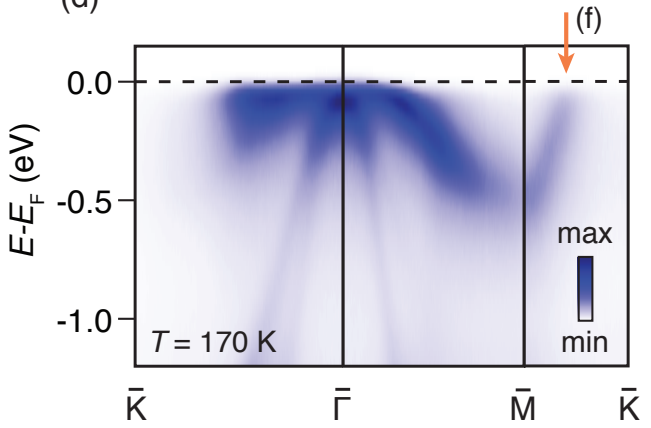

(e)

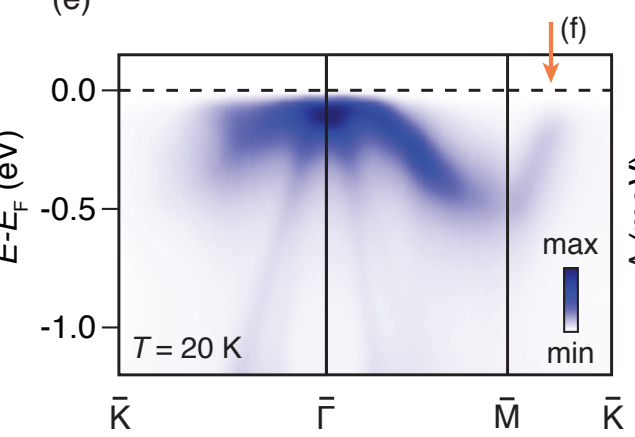

(f)

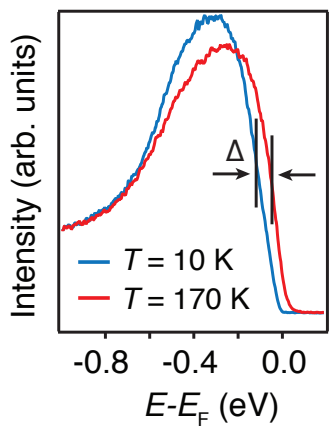

(g)

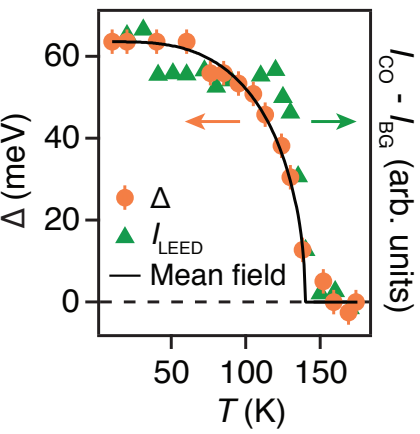

FIG. 2. Charge density-wave order of monolayer $\mathrm{VSe}_{2}$. (a,b) Low energy electron diffraction of (a) bilayer Graphene/SiC and (b) following additional growth of $\mathrm{ML}_{-\mathrm{VSe}_{2}}(T=170 \mathrm{~K}, E=150 \mathrm{eV})$. (c) Difference image of ML-VSe 2 LEED $(E=100 \mathrm{eV})$ at $T=40 \mathrm{~K}$ and $T=200 \mathrm{~K}$, revealing the emergence of additional charge-order peaks at low temperature, as shown magnified and with enhanced contrast in the inset. (d,e) Corresponding evolution of the measured electronic structure $(h \nu=21.2 \mathrm{eV})$ from (d) $T=170 \mathrm{~K}$ to (e) $T=20 \mathrm{~K}$, revealing the opening of a charge-density wave gap at the Fermi level. (f) This is clearly evident in EDCs at $k_{F}$ along the $\overline{\mathrm{M}}-\overline{\mathrm{K}}$ direction (at the position marked in (d,e)). (g) The temperature-dependent shift of the leading edge midpoint (LEM) of these EDCs, $\Delta$ (left), is in good agreement with the intensity-dependence of the charge-order superstructure from LEED, plotted as $I_{\mathrm{CO}}-I_{\mathrm{BG}}$ to take account of temperature-dependent background variations, where the charge-order and background regions are defined in (c). The solid line in (g) shows a fit to a semi-phenomenological mean-field form for the gap opening: $\Delta(T) \propto \tanh \left(C \sqrt{\frac{T_{c}}{T}-1}\right)$ where $C$ is a constant.

strictly two-dimensional. Our measured Fermi surface (Fig. 1(i)) exhibits nearly-triangular hole-like Fermi surfaces around the corner $\bar{K}$-points of the $2 \mathrm{D}$ Brillouin zone. This is most similar to the bulk Fermi surface for the $k_{z}=0$ plane. Moving below the Fermi level, the constant energy contours evolve into closed electronlike pockets centered around the $\overline{\mathrm{M}}$-points, similar to the bulk Fermi surface within the $k_{z}=\pi / c$ plane. Our DFT calculations (Fig. 1(j)) indicate that the van Hove singularity that marks the transition between these cases is situated within $\sim 20 \mathrm{meV}$ of the Fermi level. From our measured dispersions along $\bar{\Gamma}-\overline{\mathrm{K}}$, we find experimentally that this van Hove singularity is located at a slightly higher binding energy of $45 \mathrm{meV}$. This may reflect a slight electron doping of our monolayer films due to charge transfer from the substrate. Nonetheless, its proximity to the Fermi level raises the tantalising prospect to drive a Lifshitz transition in monolayer $\mathrm{VSe}_{2}$ by moder- ate charge carrier doping either via chemical substitution or transistor-style gating approaches.

The $\pi$-states of the underlying bilayer graphene substrate are also visible in our measurements, situated on the vertices of a hexagon in Fig. 1(i) where they are evident as small electron pockets due to the residual electron doping of our epitaxial graphene samples. The Brillouin zone of the $\mathrm{VSe}_{2}$ lattice is in good rotational alignment with the graphene one but is smaller. This points to a weak coupling between the substrate and the overlayer such that strain is not coupled into the asgrown film. This is consistent with our reflection highenergy electron diffraction (RHEED, Fig. 1(g)) measurements which indicate an in-plane lattice constant of our monolayer $\mathrm{VSe}_{2}$ of $a=3.31 \pm 0.05 \AA$, in good agreement with that of bulk $\mathrm{VSe}_{2}$. This is further supported by our low-energy electron diffraction (LEED) measurements (Fig. 2(a-c)). Sharp Bragg spots and a well-defined 
moiré superstructure, arising due to the lattice mismatch between graphene and $\mathrm{SiC}$, are observed for our bilayer graphene/SiC substrates (Fig. 2(a)). As is typical for epitaxial TMD monolayers, ${ }^{28,39,40}$ the Bragg spots of our $\mathrm{VSe}_{2}$ are rather elongated in the azimuthal direction (Fig. 2(b)), indicating some rotational disorder due to the weak substrate-overlayer interaction. Their intensity is, however, strongly peaked in the direction parallel to the Bragg spots of the graphene lattice, indicating that they retain a good overall epitaxial registry.

At low temperatures, a new set of features appear in our LEED measurements (Fig. 2(c)), labelled 'CO' in the magnified image. These show a similar angular spread as for the $\mathrm{VSe}_{2}$ Bragg spots discussed above, but are much weaker in overall intensity. We assign these as chargeorder peaks indicative of a CDW instability persisting to the monolayer limit of $\mathrm{VSe}_{2}$, as further supported below. The location of the new features that we observe in LEED is consistent with the $\bar{\Gamma}_{\{11\}}$ family of charge order spots for an ordering wavevector $\mathbf{q}_{\mathrm{CDW}}=(1 / 4,1 / 4)$, retaining the in-plane periodicity of the bulk CDW order, ${ }^{15,30,41}$ where the $\bar{\Gamma}_{\{01\}}$ spots would be shadowed by the electron gun in our LEED optics. Although this is certainly plausible, further measurements, for example scanning tunnelling microscopy or x-ray scattering, will be required to unambiguously classify $\mathbf{q C D W}_{\mathrm{CD}}$ here. Irrespective of the precise ordering vector, we show below how this CDW has a pronounced effect on the low-energy electronic structure of monolayer $\mathrm{VSe}_{2}$.

At the Fermi crossing along the $\bar{M}-\bar{K}$ direction, a pronounced CDW gap opens, evident as a shift of $\Delta=64 \pm 5 \mathrm{meV}$ of the leading edge midpoint (LEM) of EDCs measured at $k_{F}$ of the normal state Fermi surface (Fig. 2(f)). Monitoring this leading-edge shift as a function of temperature (Fig. 2(g)), we observe a gap opening that closely follows the intensity of the charge-order superstructure peak that we observe in LEED. These both onset at $T_{c}=140 \pm 5 \mathrm{~K}$, providing unambiguous evidence that charge-density wave order not only persists to, but is strengthened in, the monolayer limit of $\mathrm{VSe}_{2}$. This is consistent with transport studies of few-layer $\mathrm{VSe}_{2}$ obtained by liquid exfoliation, ${ }^{32}$ as well as the very recent observation of an increasing $T_{c}$ with decreasing material thickness extracted from scanning tunnelling microscopy measurements of mechanically exfoliated few-layer samples. $^{33}$

The CDW gap opening is reasonably described by a semi-phenomenological mean-field functional form (Fig. 2(g)). For a two-dimensional system such as this, fluctuations would be expected to reduce the temperature at which long-range order sets in. Given the relatively large linewidths of our measured EDCs (see Methods), we cannot conclusively say whether these lead to deviations from a mean-field form for the gap opening in close vicinity to $T_{\mathrm{c}}$. Irrespective, the clear spectroscopic gap opening that we observe here at $140 \pm 5 \mathrm{~K}$ points to (a) $T=180 \mathrm{~K}$

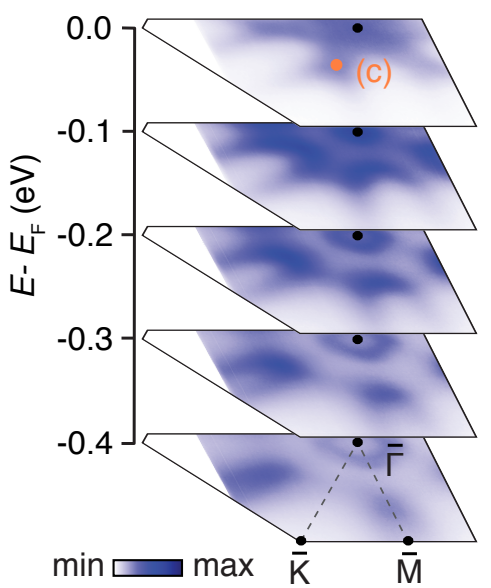

(b) $T=25 \mathrm{~K}$

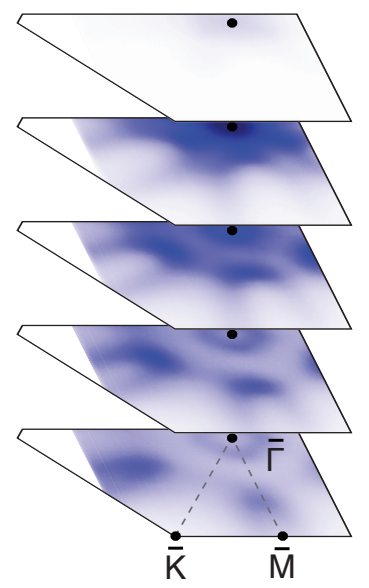

(d)

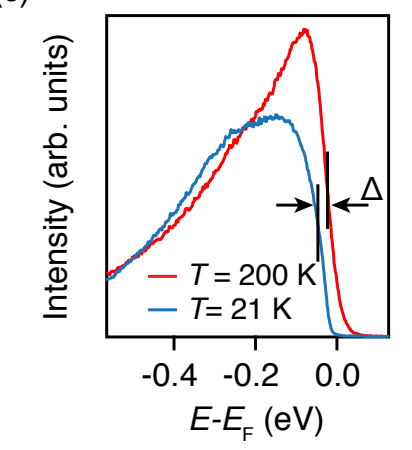

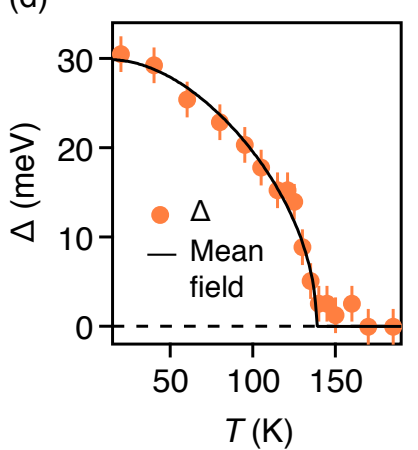

FIG. 3. Momentum-dependent CDW gaps. Fermi surface of monolayer $\mathrm{VSe}_{2}$ measured $(h \nu=21.2 \mathrm{eV})$ at (a) $T=$ $180 \mathrm{~K}$ and (b) $T=20 \mathrm{~K}$. (c) Corresponding EDCs at the tip of the $\overline{\mathrm{K}}$-centered Fermi surface (orange dot in (a)) and (d) temperature-dependent gap opening extracted from the LEM shift of such EDCs, together with a mean-field fit (solid line).

a robust enhancement of the $T_{\mathrm{c}}$ for monolayer $\mathrm{VSe}_{2}$ as compared to the bulk $T_{\mathrm{c}}$ of $110 \mathrm{~K}$.

Our measured low-temperature gap magnitude and CDW transition temperature yield a value of $2 \Delta / k_{\mathrm{B}} T_{\mathrm{c}} \approx$ 10 which significantly exceeds the value of 3.52 that would be expected for a weak-coupling mean-field instability. In agreement with this, Figs. 3(a,b) show how the closed Fermi pockets around the $\overline{\mathrm{K}}$-points that are evident at high temperature lose almost all of their spectral weight below $T_{c}$. This indicates that not only the well-nested flat portions of Fermi surface near the $\overline{\mathrm{M}}-\overline{\mathrm{K}}$ line become gapped by the CDW, but rather the entire Fermi surface opens a CDW gap. Indeed, EDCs taken at the apex of the Fermi surface along $\bar{\Gamma}-\overline{\mathrm{K}}$ (Fig. 3(c)) indicate a similar temperature-dependent gap opening (Fig. 3(d)) to that discussed above for the Fermi crossing along $\overline{\mathrm{M}}-\overline{\mathrm{K}}$, albeit with a smaller gap magnitude of $\Delta=30 \pm 5 \mathrm{meV}$.

This is in stark contrast to bulk $\mathrm{VSe}_{2}$, where gaps are 
(a)

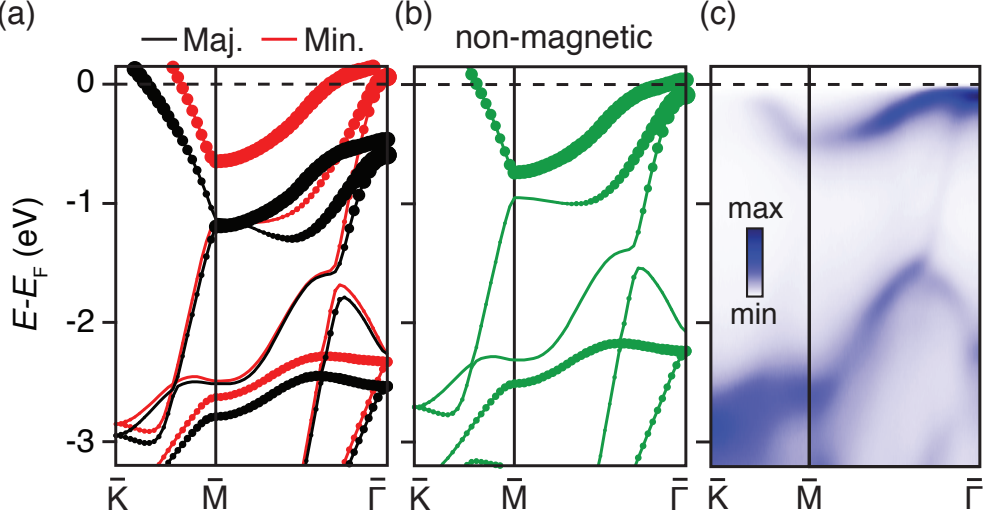

(d)

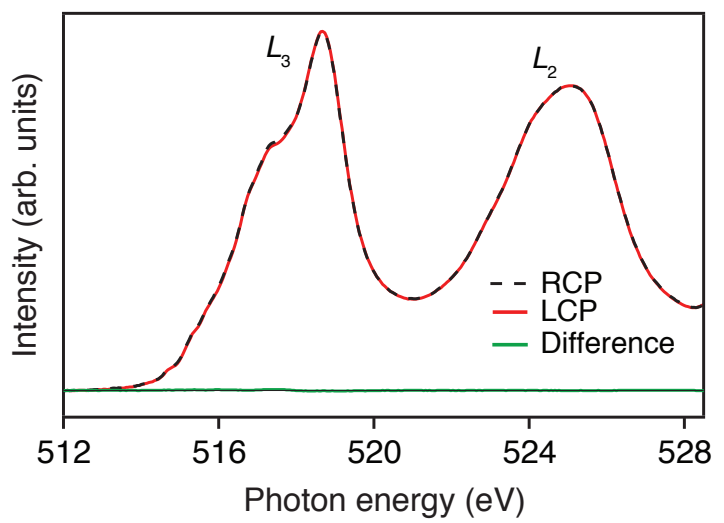

FIG. 4. Absence of ferromagnetic order. Electronic structure of monolayer $\mathrm{VSe}_{2}$ from (a) a spin-polarised and (b) a nonmagnetic DFT calculation. The calculations are projected onto the V $3 d$ orbital character (size of the points), and the majority and minority spin components are shown respectively as red and black colouring in (a). (c) Equivalent experimental electronic structure, which is consistent only with the non-magnetic calculation. (d) $\mathrm{V} L_{2,3}$-edge x-ray absorption measurements performed using left-circularly polarised (LCP) and right-circularly polarised (RCP) light in an applied magnetic field of 9 T $(T=100 \mathrm{~K}$, red and black lines, respectively), and corresponding difference signal (green), show the absence of XMCD, further ruling out ferromagnetic order.

thought to only open over small portions of the Fermi surface which are well-nested. ${ }^{31}$ In the monolayer, we find that the flat portions of Fermi surface have a nesting vector along $\mathbf{a}^{*}$ of $\mathbf{q}_{\text {nest }}=0.54 \pm 0.04 \AA^{-1}$, which is close to the ordering wavevector of the CDW discussed above. Nonetheless, while this may help to enhance the CDW order, the large overall gap magnitude, in particular persisting to the tips of the Fermi surface where there is negligible nesting, instead points to a strong electronphonon coupling as the main driver of the CDW instability here. Similarly, we note that the apparently even better Fermi surface nesting along the one-dimensional directions at $30^{\circ}$ rotation from the $\mathbf{a}^{*}$ direction that is evident in Fig. 1(i,j)) does not appear to dominate the CDW ordering vector here, further confirming that nesting is not the driving force of the $\mathrm{CDW}$ in monolayer $\mathrm{VSe}_{2}$. Consistent with this, we also find a fully- or near-fully gapped CDW phase from our measurements on HOPG substrates (Supplementary Fig. S1), with a comparable critical temperature $\left(T_{c}=134 \pm 5 \mathrm{~K}\right)$ to the measurements shown here for graphene substrates.

Finally, we return to the question of whether monolayer $\mathrm{VSe}_{2}$ hosts an intrinsic ferromagnetic state. Indeed, consistent with previous calculations, ${ }^{32,34}$ our DFT of single-layer $\mathrm{VSe}_{2}$ indicates that a ferromagnetic ground state is the lowest energy configuration. Comparing the calculated electronic structure in the ferromagnetic state (Fig. 4(a)) with equivalent non-magnetic calculations (Fig. 4(b)), the ferromagnetism can be seen to dominantly affect the V-derived states, inducing a splitting of $\sim 500 \mathrm{meV}$ between the spin majority and minority bands near the Fermi level. Such a pronounced ex- change splitting is inconsistent with our experimentallymeasured dispersion (Fig. 4(c)), where only a single $d$ band dispersion is observed. Indeed, the overall band dispersions observed experimentally are entirely consistent with the non-magnetic calculation apart from a small difference in doping and an overestimation of the bandwidth of the near- $E_{\mathrm{F}}$ states by a factor of $\sim 1.4$, likely arising due to moderate electronic correlations associated with the $\mathrm{V} 3 d$ orbitals which are not captured in DFT.

A lack of ferromagnetism here is further supported by measurements of $\mathrm{V} L_{2,3}$-edge x-ray magnetic circular dichroism (XMCD) from our monolayer $\mathrm{VSe}_{2}$ samples, shown in Fig. 4(d). XMCD is a sensitive elementspecific probe of magnetic order. Despite this, we find negligible dichroism either when measured in an applied magnetic field of $9 \mathrm{~T}$ or when measured in remanence, and at temperatures of $300 \mathrm{~K}$ or $100 \mathrm{~K}$ (Fig. 4(d)). This directly rules out any ferromagnetic order of monolayer $\mathrm{VSe}_{2}$ down to $T=100 \mathrm{~K}$, in stark contrast to the recent report of robust ferromagnetism at room temperature in this system. ${ }^{35}$ The absence of an exchange splitting in the electronic structure, discussed above, further allows us to exclude that ferromagnetism develops down to our lowest ARPES measurement temperature of $T=10 \mathrm{~K}$.

It remains an open question why our DFT calculations, consistent with previous literature, ${ }^{32,34}$ dramatically over-estimate the tendency for ferromagnetism in monolayer $\mathrm{VSe}_{2}$. We speculate that phase fluctuations in the two-dimensional limit may play a more important role for the ferromagnetic state here, renormalising its energy scale to below that at which the CDW takes over as the dominant instability. The consequent full gap- 
ping of the Fermi surface that we observe would then remove the driving force for a Stoner-type ferromagnetic order. ${ }^{42}$ This suggests an appealing potential for engineering competition between disparate ground states in ML-VSe 2 and other TMDs. These may be tuneable by, for example, charge carrier doping or promoting magnetic order via proximity effects. Beyond this, our study demonstrates how charge order can be readily manipulated in two-dimensional materials, here not only occurring with a significantly increased $T_{c}$ as compared to the bulk, but also exhibiting signatures of a much strongercoupling instability in the single-layer limit of $\mathrm{VSe}_{2}$.

\section{Methods.}

Molecular-beam epitaxy: Our films are grown using molecular-beam epitaxy on epitaxial bilayer graphene/SiC as well as highly oriented pyrolytic graphite (HOPG) substrates in a UHV chamber with a base pressure of $\sim 1 \times 10^{-10}$ mbar. A high temperature effusion cell with $4 \mathrm{~N}$ pure $\mathrm{V}$ and a valved cracker cell with $5 \mathrm{~N}$ pure Se are used as sources.

For a typical growth, the substrate is first annealed to $550^{\circ} \mathrm{C}$ for 60 minutes before cooling to a growth temperature of $300^{\circ} \mathrm{C}$ (thermocouple temperature). During growth, the $\mathrm{V}$ flux is maintained at a beam-equivalent pressure (BEP) of $\sim 6 \times 10^{-10}$ mbar, whereas approximately an order of magnitude greater Se flux is used. Our growth rate of $1 \mathrm{ML} /$ hour is dictated by the $\mathrm{V}$ flux due to the near unity sticking coefficient of $\mathrm{V}$ at the growth temperature. As growth progresses, the streaky RHEED patterns visible from both graphene and HOPG substrates gradually disappear and the new $\mathrm{VSe}_{2}$ streaks begin to appear (see Supplementary Fig. S2). These new patterns become strong and streaky towards the end of the growth, confirming the flat morphology of the monolayer $\mathrm{VSe}_{2}$ surface. This, as well as a nearly-uniform ML surface coverage, are confirmed by atomic force microscopy (Supplementary Fig. S3). Some samples were capped with a protective Se layer after growth. For these, the samples are cooled down to below $35^{\circ} \mathrm{C}$ before depositing an amorphous layer of Se for 10 minutes. The Se BEP is maintained at $\sim 8 \times 10^{-7}$ mbar, which provides an estimated deposition rate of $1 \mathrm{~nm} / \mathrm{min}$.

For substrates, fresh HOPG surfaces were exfoliated in atmosphere and straightaway transferred into a vacuum load lock. For graphene synthesis, we use a variation of a widely demonstrated technique for epitaxial bilayer graphene growth: ${ }^{43} 3 \times 7 \mathrm{~mm}^{2}$ wafers of $n$-type $6 \mathrm{H}-\mathrm{SiC}(0001)$ are first cleaned using acetone and isopropanol. Two such substrates are then placed one on top of another with the Si terminated surfaces facing each other and a $0.12 \mathrm{~mm}$ thick Ta spacer between them on both ends. This 'sandwich' is then loaded into a vacuum chamber and annealed at $600^{\circ} \mathrm{C}$ for 6 hours by direct current heating. After this, the substrates are further heated to $1500^{\circ} \mathrm{C}$ for 7 minutes to produce bilayer graphene layers. These yield sharp Bragg spots and well-defined moiré patterns in LEED measurements (Fig. 2(a)).

Angle-resolved photoemission: Following growth, our samples were transferred in situ to a lab-based ARPES setup. ARPES measurements were performed using a high-intensity He lamp ( $h \nu=21.2 \mathrm{eV}, p$-polarisation) and a SPECS Phoibos 225 hemispherical electron analyzer, at temperatures between 10 and $180 \mathrm{~K}$. LEED measurements were performed in the same system, using a SPECS ErLEED 150. We note that the measured linewidths of our EDCs are significantly higher than the experimental energy resolution, in common with other ARPES studies of monolayer TMDs. This could reflect impurity scattering, finite domain-size effects, and the effects of rotational disorder. The resultant broadening can lead to an underestimate of the CDW gap size from the LEM shift, although we stress that this analysis method still yields a robust value of $T_{\mathrm{c}}$.

Monolayer $\mathrm{VSe}_{2}$ samples were also measured at the $\mathrm{I} 05$ beamline of Diamond Light Source, UK, using photon energies of $92.5 \mathrm{eV}$ and $110 \mathrm{eV}$, and a Scienta R4000 analyzer. The measurements shown in Fig. 1(i) are the sum of Fermi surfaces recorded using left- and right-circularly polarised light. The samples were Se-capped following growth, as described above, and transferred through air, before being decapped by annealing to $\sim 230^{\circ} \mathrm{C}$ for 15 minutes in a preparation chamber connected to the I05 ARPES chamber. Characterisation by LEED and ARPES in our home system indicates that the capping, air exposure, and de-capping procedure leads to no noticable changes in the measured electronic structure. In addition, ARPES measurements of bulk $\mathrm{VSe}_{2}$ crystals $^{44}$ were performed using the I05 beamline as well as the CASSIOPEE beamline of the SOLEIL synchrotron, France, using Scienta R4000 electron analyzers. The data shown in Fig. $1(\mathrm{~b}, \mathrm{c})$ is the sum of measurements performed using $p$ and $s$-polarised light, while $p$-polarised light is used for the Fermi surfaces shown in Fig. 1(d). The samples were cleaved in situ and measured at a temperature of $170 \mathrm{~K}$.

Calculations: The electronic structure of monolayer $\mathrm{VSe}_{2}$ was calculated within density-functional theory. The DFT calculations were performed using the Wien $2 \mathrm{k}$ package ${ }^{45}$ utilizing the linearized augmented plane wave (LAPW) method and the Perdew Burke and Ernzerhof (PBE) exchange-correlation functional. ${ }^{46} \mathrm{~A}$ separation of $18 \AA$ between $\mathrm{V}$ layers in our supercell (corresponding to a vacuum gap of $14.85 \AA$ was found to be sufficient to ensure our calculations were representative of an isolated monolayer. We employed a $14 \times 14 \times 2 \mathrm{k}$-point mesh, and the internal lattice parameters were optimised until the total force is less than $2 \mathrm{mRy} / \mathrm{a} . u$.

X-ray magnetic circular dichroism: XMCD measurements were performed in total electron yield (TEY) mode from Se-capped samples at the ID32 beamline of the ESRF synchrotron. The sample was mounted with the incoming $\mathrm{x}$-ray beam at $55^{\circ}$ to the surface normal. The $\mathrm{V} L_{2,3}$-edge X-ray absorption was clearly visible in TEY mode, but was only very weakly evident when measuring in fluorescence mode due to the monolayer thickness. XMCD measurements were performed first at room temperature in an applied magnetic field of $9 \mathrm{~T}$ and then in remanence. This measurement cycle was repeated after cooling to $100 \mathrm{~K}$. All measurements yielded a consistent result of negligible dichroism.

Acknowledgements. We thank Andrew Huxley for useful discussions. We gratefully acknowledge support from The Leverhulme Trust (Grant Nos. RL-2016-006 and PLP-2015-144), The Royal Society, The Engineering 
and Physical Sciences Research Council, UK, for support under grant Nos. EP/I031014/1, EP/M023958/1, $\mathrm{EP} / \mathrm{P} 020151 / 1$, and $\mathrm{EP} / \mathrm{M} 028771 / 1$, and the International Max-Planck Partnership for Measurement and Observation at the Quantum Limit. OJC and $\mathrm{KU}$ acknowledge EPSRC for PhD studentship support through grant Nos. EP/K503162/1 and EP/L015110/1. IM acknowledges $\mathrm{PhD}$ studentship support from the IMPRS for the Chemistry and Physics of Quantum Materials. LBD acknowledges studentship support from EPSRC and the Science and Technology Facilities Council (UK). We thank Diamond Light Source for access to beamline I05 (proposal number SI19771-1), SOLEIL for access to the CASSIOPEE beamline, and the ESRF for access to the ID32 beamline, that all contributed to the results presented here, and we are grateful to Andreas Frisk for assistance with the XMCD measurements.

Supporting Information: The Supporting Information is available free of charge on the ACS Publications website at DOI: xxxxxxxxxxxxxxxxxxxx.

Supporting Figures 1-3, describing equivalent measurements to those shown in the main text for samples grown on HOPG substrates and additional growth and materials characterisation from reflection high-energy electron diffraction and atomic force microscopy.

Note: The authors declare no competing financial interests.

* These authors contributed equally

$\dagger$ Current address: Department of Physics, Birla Institute of Technology, Mesra 835215, Ranchi, India

‡ philip.king@st-andrews.ac.uk

[1] Mak, K. F.; Lee, C.; Hone, J.; Shan, J.; Heinz, T. F. Atomically Thin $\mathrm{MoS}_{2}$ : A New Direct-Gap Semiconductor. Phys. Rev. Lett. 2010, 105, 136805.

[2] Mannhart, J.; Schlom, D. G. Oxide Interfaces - an Opportunity for Electronics. Science 2010, 327, 1607-1611.

[3] Hwang, H. Y.; Iwasa, Y.; Kawasaki, M.; Keimer, B.; Nagaosa, N.; Tokura, Y. Emergent Phenomena at Oxide Interfaces. Nature Mater. 2012, 11, 103-113.

[4] Wang, Q. H.; Kalantar-Zadeh, K.; Kis, A.; Coleman, J. N.; Strano, M. S. Electronics and Optoelectronics of Two-dimensional Transition Metal Dichalcogenides. Nat Nano 2012, 7, 699-712.

[5] Geim, A. K.; Grigorieva, I. V. Van der Waals Heterostructures. Nature 2013, 499, 419-425.

[6] Xu, X.; Yao, W.; Xiao, D.; Heinz, T. F. Spin and Pseudospins in Layered Transition Metal Dichalcogenides. Nat. Phys. 2014, 10, 343-350.

[7] Ajayan, P.; Kim, P.; Banerjee, K. Two-dimensional Van Der Waals Materials. Phys. Today 2016, 69, 38-44.

[8] Chhowalla, M.; Shin, H. S.; Eda, G.; Li, L.-J.; Loh, K. P.; Zhang, H. The Chemistry of Two-dimensional Layered Transition Metal Dichalcogenide Nanosheets. Nat. Chem.
2013, 5, 263-275.

[9] Riley, J. M.; Mazzola, F.; Dendzik, M.; Michiardi, M.; Takayama, T.; Bawden, L.; Granerød, C.; Leandersson, M.; Balasubramanian, T.; Hoesch, M.; Kim, T. K.; Takagi, H.; Meevasana, W.; Hofmann, P.; Bahramy, M. S.; Wells, J. W.; King, P. D. C. Direct Observation of Spin-polarized Bulk Bands in an Inversionsymmetric Semiconductor. Nat. Phys. 2014, 10, 835839.

[10] Lu, J. M.; Zheliuk, O.; Leermakers, I.; Yuan, N. F. Q.; Zeitler, U.; Law, K. T.; Ye, J. T. Evidence for Twodimensional Ising Superconductivity in Gated $\mathrm{MoS}_{2}$. Science 2015, 350, 1353-1357.

[11] Riley, J. M.; Meevasana, W.; Bawden, L.; Asakawa, M.; Takayama, T.; Eknapakul, T.; Kim, T. K.; Hoesch, M.; Mo, S.-K.; Takagi, H.; Sasagawa, T.; Bahramy, M. S.; King, P. D. C. Negative Electronic Compressibility and Tunable Spin Splitting in $\mathrm{WSe}_{2}$. Nat Nano 2015, 10, 1043-1047.

[12] Kogar, A.; Rak, M. S.; Vig, S.; Husain, A. A.; Flicker, F.; Joe, Y. I.; Venema, L.; MacDougall, G. J.; Chiang, T. C.; Fradkin, E.; Wezel, J. v.; Abbamonte, P. Signatures of Exciton Condensation in a Transition Metal Dichalcogenide. Science 2017, 358, 1314-1317.

[13] Bahramy, M. S.; Clark, O. J.; Yang, B.-J.; Feng, J.; Bawden, L.; Riley, J. M.; Marković, I.; Mazzola, F.; Sunko, V.; Biswas, D.; Cooil, S. P.; Jorge, M.; Wells, J. W.; Leandersson, M.; Balasubramanian, T.; Fujii, J.; Vobornik, I.; Rault, J. E.; Kim, T. K.; Hoesch, M.; Okawa, K.; Asakawa, M.; Sasagawa, T.; Eknapakul, T.; Meevasana, W.; King, P. D. C. Ubiquitous Formation of Bulk Dirac Cones and Topological Surface States from a Single Orbital Manifold in Transition-metal Dichalcogenides. Nat. Mater. 2018, 17, 21.

[14] Wilson, J. A.; Di Salvo, F. J.; Mahajan, S. ChargeDensity Waves in Metallic, Layered, Transition-Metal Dichalcogenides. Phys. Rev. Lett. 1974, 32, 882-885.

[15] Tsutsumi, K. X-ray-diffraction Study of the Periodic Lattice Distortion Associated with a Charge-density Wave in $1 T-\mathrm{VSe}_{2}$. Phys. Rev. B 1982, 26, 5756-5759.

[16] Johannes, M. D.; Mazin, I. I.; Howells, C. A. Fermisurface Nesting and the Origin of the Charge-density Wave in $\mathrm{NbSe}_{2}$. Phys. Rev. B 2006, 73, 205102.

[17] Borisenko, S. V.; Kordyuk, A. A.; Yaresko, A. N.; Zabolotnyy, V. B.; Inosov, D. S.; Schuster, R.; Büchner, B.; Weber, R.; Follath, R.; Patthey, L.; Berger, H. Pseudogap and Charge Density Waves in Two Dimensions. Phys. Rev. Lett. 2008, 100, 196402.

[18] Bawden, L.; Cooil, S. P.; Mazzola, F.; Riley, J. M.; Collins-McIntyre, L. J.; Sunko, V.; Hunvik, K. W. B.; Leandersson, M.; Polley, C. M.; Balasubramanian, T.; Kim, T. K.; Hoesch, M.; Wells, J. W.; Balakrishnan, G.; Bahramy, M. S.; King, P. D. C. Spin-valley Locking in the Normal State of a Transition-metal Dichalcogenide Superconductor. Nat. Commun. 2016, 7, 11711.

[19] Morosan, E.; Zandbergen, H. W.; Dennis, B. S.; Bos, J. W. G.; Onose, Y.; Klimczuk, T.; Ramirez, A. P.; Ong, N. P.; Cava, R. J. Superconductivity in $\mathrm{Cu}_{x} \mathrm{TiSe}_{2}$. Nat. Phys. 2006, 2, 544-550.

[20] Kiss, T.; Yokoya, T.; Chainani, A.; Shin, S.; Hanaguri, T.; Nohara, M.; Takagi, H. Charge-ordermaximized Momentum-dependent Superconductivity. Nat. Phys. 2007, 3, 720-725.

[21] Rahn, D. J.; Hellmann, S.; Kalläne, M.; Sohrt, C.; 
Kim, T. K.; Kipp, L.; Rossnagel, K. Gaps and Kinks in the Electronic Structure of the Superconductor $2 H$ $\mathrm{NbSe}_{2}$ from Angle-resolved Photoemission at $1 \mathrm{~K}$. Phys. Rev. B 2012, 85, 224532.

[22] Li, L. J.; ÓFarrell, E. C. T.; Loh, K. P.; Eda, G.; Özyilmaz, B.; Castro Neto, A. H. Controlling Many-body States by the Electric-field Effect in a Two-dimensional Material. Nature 2015, 529, 185-189.

[23] Yang, J.; Wang, W.; Liu, Y.; Du, H.; Ning, W.; Zheng, G.; Jin, C.; Han, Y.; Wang, N.; Yang, Z.; Tian, M.; Zhang, Y. Thickness Dependence of the Charge-density-wave Transition Temperature in $\mathrm{VSe}_{2}$. Appl. Phys. Lett. 2014, 105, 063109.

[24] Xi, X.; Zhao, L.; Wang, Z.; Berger, H.; Forró, L.; Shan, J.; Mak, K. F. Strongly Enhanced Charge-density-wave Order in Monolayer $\mathrm{NbSe}_{2}$. Nat Nano 2015, 10, 765-769.

[25] Yu, Y.; Yang, F.; Lu, X. F.; Yan, Y. J.; Cho, Y.-H.; Ma, L.; Niu, X.; Kim, S.; Son, Y.-W.; Feng, D.; Li, S.; Cheong, S.-W.; Chen, X. H.; Zhang, Y. Gate-tunable Phase Transitions in Thin Flakes of $1 T-\mathrm{TaS}_{2}$. Nat Nano 2015, 10, 270-276.

[26] Yoshida, M.; Suzuki, R.; Zhang, Y.; Nakano, M.; Iwasa, Y. Memristive Phase Switching in Twodimensional $1 T-\mathrm{TaS}_{2}$ Crystals. Sci. Adv. 2015, 1, e1500606.

[27] Chen, P.; Chan, Y.-H.; Fang, X.-Y.; Zhang, Y.; Chou, M.-Y.; Mo, S.-K.; Hussain, Z.; Fedorov, A.-V.; Chiang, T.-C. Charge Density Wave Transition in Singlelayer Titanium Diselenide. Nat. Commun. 2015, 6, 8943.

[28] Ugeda, M. M.; Bradley, A. J.; Zhang, Y.; Onishi, S.; Chen, Y.; Ruan, W.; Ojeda-Aristizabal, C.; Ryu, H.; Edmonds, M. T.; Tsai, H.-Z.; Riss, A.; Mo, S.-K.; Lee, D.; Zettl, A.; Hussain, Z.; Shen, Z.-X.; Crommie, M. F. Characterization of Collective Ground States in Single-layer $\mathrm{NbSe}_{2}$. Nat. Phys. 2016, 12, 92-97.

[29] Ryu, H.; Chen, Y.; Kim, H.; Tsai, H.-Z.; Tang, S.; Jiang, J.; Liou, F.; Kahn, S.; Jia, C.; Omrani, A. A.; Shim, J. H.; Hussain, Z.; Shen, Z.-X.; Kim, K.; Min, B. I.; Hwang, C.; Crommie, M. F.; Mo, S.-K. Persistent Charge-Density-Wave Order in Single-Layer TaSe $\mathrm{T}_{2}$. Nano Lett. 2018, 18, 689-694.

[30] Eaglesham, D. J.; Withers, R. L.; Bird, D. M. Chargedensity-wave Transitions in $1 T-\mathrm{VSe}_{2}$. J. Phys. C: Solid State Phys. 1986, 19, 359.

[31] Strocov, V. N.; Shi, M.; Kobayashi, M.; Monney, C.; Wang, X.; Krempasky, J.; Schmitt, T.; Patthey, L.; Berger, H.; Blaha, P. Three-Dimensional Electron Realm in $\mathrm{VSe}_{2}$ by Soft-X-Ray Photoelectron Spectroscopy: Origin of Charge-Density Waves. Phys. Rev. Lett. 2012, 109, 086401.

[32] Xu, K.; Chen, P.; Li, X.; Wu, C.; Guo, Y.; Zhao, J.; Wu, X.; Xie, Y. Ultrathin Nanosheets of Vanadium Diselenide: A Metallic Two-Dimensional Material with Ferromagnetic Charge-Density-Wave Behavior. Angew. Chem. Int. Ed. 2013, 52, 10477-10481.

[33] Pásztor, Á.; Scarfato, A.; Barreteau, C.; Giannini, E.; Renner, C. Dimensional Crossover of the Charge Density Wave Transition in Thin Exfoliated $\mathrm{VSe}_{2}$. $2 D$ Mater. 2017, 4, 041005.

[34] Ma, Y.; Dai, Y.; Guo, M.; Niu, C.; Zhu, Y.; Huang, B. Evidence of the Existence of Magnetism in Pristine $\mathrm{VX}_{2}$ Monolayers ( $\mathrm{X}=\mathrm{S}, \mathrm{Se})$ and Their Strain-Induced Tunable Magnetic Properties. ACS Nano 2012, 6, 1695-
1701.

[35] Bonilla, M.; Kolekar, S.; Ma, Y.; Diaz, H. C.; Kalappattil, V.; Das, R.; Eggers, T.; Gutierrez, H. R.; Phan, M.H.; Batzill, M. Strong Room-temperature Ferromagnetism in $\mathrm{VSe}_{2}$ Monolayers on Van der Waals Substrates. Nat. Nanotechnol. 2018, 13, 289-293.

[36] Gong, C.; Li, L.; Li, Z.; Ji, H.; Stern, A.; Xia, Y.; Cao, T.; Bao, W.; Wang, C.; Wang, Y.; Qiu, Z. Q.; Cava, R. J.; Louie, S. G.; Xia, J.; Zhang, X. Discovery of Intrinsic Ferromagnetism in Two-dimensional Van der Waals Crystals. Nature 2017, 546, 265-269.

[37] Huang, B.; Clark, G.; Navarro-Moratalla, E.; Klein, D. R.; Cheng, R.; Seyler, K. L.; Zhong, D.; Schmidgall, E.; McGuire, M. A.; Cobden, D. H.; Yao, W.; Xiao, D.; Jarillo-Herrero, P.; Xu, X. Layerdependent Ferromagnetism in a Van der Waals Crystal down to the Monolayer Limit. Nature 2017, 546, 270-273.

[38] Zunger, A.; Freeman, A. J. Electronic Structure of $1 T$ $\mathrm{VSe}_{2}$. Phys. Rev. B 1979, 19, 6001.

[39] Zhang, Y.; Chang, T.-R.; Zhou, B.; Cui, Y.-T.; Yan, H.; Liu, Z.; Schmitt, F.; Lee, J.; Moore, R.; Chen, Y.; Lin, H.; Jeng, H.-T.; Mo, S.-K.; Hussain, Z.; Bansil, A.; Shen, Z.$\mathrm{X}$. Direct Observation of the Transition from Indirect to Direct Bandgap in Atomically Thin Epitaxial $\mathrm{MoSe}_{2}$. Nat Nano 2014, 9, 111-115.

[40] Jiao, L.; Liu, H. J.; Chen, J. L.; Yi, Y.; Chen, W. G.; Cai, Y.; Wang, J. N.; Dai, X. Q.; Wang, N.; Ho, W. K.; Xie, M. H. Molecular-beam Epitaxy of Monolayer $\mathrm{MoSe}_{2}$ : Growth Characteristics and Domain Boundary Formation. New J. Phys. 2015, 17, 053023.

[41] Zhang, D.; Ha, J.; Baek, H.; Chan, Y.-H.; Natterer, F. D.; Myers, A. F.; Schumacher, J. D.; Cullen, W. G.; Davydov, A. V.; Kuk, Y.; Chou, M. Y.; Zhitenev, N. B.; Stroscio, J. A. Strain Engineering a $4 a \times \sqrt{3}$ a Chargedensity-wave Phase in Transition-metal Dichalcogenide $1 T-\mathrm{VSe}_{2}$. Physical review materials 2017, 1, 024005.

[42] Fumega, A. O.; Pardo, V. Absence of Ferromagnetism in $\mathrm{VSe}_{2}$ Caused by Its Charge Density Wave Phase. arXiv preprint arXiv:1804.07102 2018,

[43] Yu, X.; Hwang, C.; Jozwiak, C.; Köhl, A.; Schmid, A.; Lanzara, A. New Synthesis Method for the Growth of Epitaxial Graphene. J. Electron Spectrosc. Relat. Phenom. 2011, 184, 100-106.

[44] Barua, S.; Hatnean, M. C.; Lees, M.; Balakrishnan, G. Signatures of the Kondo Effect in $\mathrm{VSe}_{2}$. Sci. Rep. 2017, 7, 10964.

[45] Blaha, P.; Schwarz, K.; Madsen, G.; Kvasnicka, D.; Luitz, J. WIEN2k, an Augmented Plane Wave + Local Orbitals Program for Calculating Crystal Properties. Technische Universität Wien, Austria. 2001.

[46] Perdew, J. P.; Burke, K.; Ernzerhof, M. Generalized Gradient Approximation Made Simple. Phys. Rev. Lett. 1996, 77, 3865-3868. 
TOC Graphic

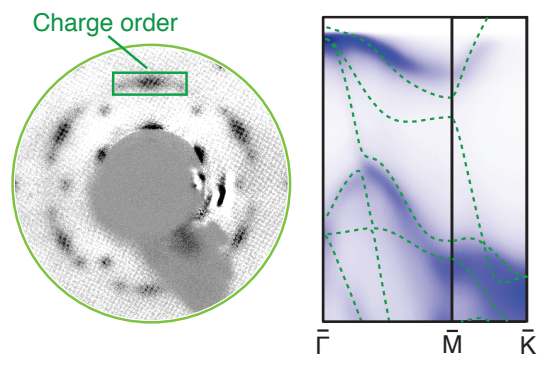

